\title{
Abordaje para nefrolitotomía percutánea. Comparación del procedimiento en un solo paso contra la secuencial con dilatadores metálicos
}

\author{
Ismael Sedano-Portillo', Gastón Ochoa-León', Clotilde Fuentes-Orozco², Leire Irusteta-Jiménez², \\ Luis Rodrigo Michel-Espinoza², Marcela Salazar-Parra², Lizbeth Cuesta-Márquez ${ }^{2}$ \\ y Alejandro González-Ojeda² \\ ${ }^{1}$ Departamento de Urología; ${ }^{2}$ Unidad de Investigación Biomédica 02. Hospital de Especialidades, Centro Médico Nacional de Occidente, Instituto \\ Mexicano del Seguro Social, Guadalajara, Jal., México
}

\begin{abstract}
Resumen
Introducción: La nefrolitotomía percutánea es un abordaje eficaz para tratar diversos tipos de cálculos renales. Se han descrito diferentes tipos de acceso, como el secuencial con dilatadores así como en un solo paso. Objetivo: Determinar diferencias entre el tiempo de exposición a rayos $X$ y los valores de la hemoglobina entre ambas técnicas. Métodos: Ensayo clínico controlado. Se incluyeron pacientes mayores de 18 años con litiasis compleja no complicada, sin infección urinaria, que se asignaron al azar a cualquiera de las dos técnicas. Las variables de respuesta se determinaron antes y 24 horas después de ambos procedimientos. Resultados: Se dividieron en dos grupos 59 pacientes, 30 con dilatación en un paso (grupo de estudio) y 29 con dilatación secuencial (grupo control). El grupo de estudio tuvo un descenso menor en los valores de hemoglobina posoperatorios que el grupo control ( 0.81 vs. $2.03 \mathrm{~g} / \mathrm{dl} ; p<0.001$ ), menor tiempo de fluoroscopia (69.6 vs. $100.62 \mathrm{~s} ; p<0.001$ ) $y$ valores séricos de creatinina posoperatorios más bajos $(0.93 \pm 0.29 \mathrm{vs} .1 .13 \pm 0.4 \mathrm{mg} / \mathrm{dl} ; p=0.039$ ). No hubo diferencias en la morbilidad posoperatoria. Conclusión: La técnica en un paso demostró mejores resultados que la dilatación secuencial.
\end{abstract}

PALABRAS CLAVE: Cálculos renales. Nefrolitotomía. Dilatación de un solo paso. Dilatación secuencial. Tiempos de fluoroscopía. Hemorragia.

\begin{abstract}
Introduction: Percutaneous nephrolithotomy is an efficient approach for treatment of different types of kidney stones. Various types of access techniques have been described like sequential dilatation and one-shot procedure. Objective: To determine the differences in time of exposure to $X$-rays and hemoglobin levels between techniques. Methods: Controlled clinical trial. Patients older than 18 years with complex/uncomplicated kidney stones, without urine infection were included. They were assigned randomly to one of the two techniques. Response variables were determined before and $24 \mathrm{~h}$ after procedures. Results: 59 patients were included: 30 underwent one-shot procedure (study-group) and 29 sequential dilatation (control-group). Baseline characteristics were similar. Study group had a lower postoperative hemoglobin decline than control group ( 0.81 vs. $2.03 \mathrm{~g} / \mathrm{dl}$, respectively; $p<0.001$ ); $X$-ray exposure time (69.6 vs. $100.62 \mathrm{~s} ; p<0.001$ ) and postoperative creatinine serum levels $(0.93 \pm 0.29 \mathrm{vs} .1 .13 \pm 0.4 \mathrm{mg} / \mathrm{dl} ; p=0.039$ ). No significant differences in postoperative morbidity were found. Conclusion: One-shot technique demonstrated better results compared to sequential dilatation.
\end{abstract}

KEY WORDS: Renal calculi. Nephrolithotomy. One-shot nephrostomy. X-ray exposure time. Hemorrhage.

\section{Correspondencia:}

Alejandro González-Ojeda

Avda. Belisario Domínguez, 1000

Col. Independencia

C.P. 44340 , Guadalajara, Jal., México

E-mail: avgail5@gmail.com

Fecha de recepción: 27-09-2016

Fecha de aceptación: 04-10-2016

avygail5@yahoo.com.mx

DOI:10.24875/GMM.17002655

Gac Med Mex. 2017;153:677-682

Contents available at PubMed

www.gacetamedicademexico.com 


\section{Introducción}

Los cálculos renales son una causa frecuente de consulta urológica. Es una condición con alta prevalencia, ya que se estima que alrededor del 10\% de la población la padecerá a lo largo de la vida ${ }^{1,2}$. Esta prevalencia se ha duplicado en los últimos 40 años, desde una incidencia anual de 54.2 por cada 100,000 habitantes en 1965 hasta 114.3 por cada 100,000 habitantes en 2005. Es más común en los hombres, pero la diferencia entre ambos sexos ha disminuido con el tiempo. Tiene un pico de incidencia entre la cuarta y la sexta décadas de la vida. La prevalencia aumenta en climas áridos, calientes y $\operatorname{secos}^{1,3}$.

La nefrolitotomía percutánea (NLP) es un procedimiento que consiste en la realización de un trayecto a través de la piel y el parénquima renal para alcanzar el sistema colector. Por medio de este trayecto se realiza la litotripsia o se pueden extraer los cálculos usando pinzas especiales (con previa nefroscopia). La NLP se ha convertido en la principal indicación para litos coraliformes, semicoraliformes de $1-2 \mathrm{~cm}^{4}$. Las principales complicaciones menores observadas en la NLP son la necesidad de transfusión (11.2-17.5\%), la fiebre (21-32\%) y el urinoma (0-1\%); y las complicaciones mayores pueden ser lesión cólica $(0.2-0.8 \%)$ o pulmonar $(0.0-3.1 \%)$, sepsis $(0.3-4.7 \%)$ y muerte $(0.0-$ $0.3 \%)$. A pesar de que la comorbilidad del paciente aumenta el riesgo de complicaciones, la mayoría de estas se resuelven de manera conservadora o por procedimientos mínimamente invasivos, presentando una evolución posquirúrgica normal en el $76.7 \%$ de los casos $^{1,5-7}$.

Dentro de los tipos de abordajes para la NLP, el tradicional consiste en la dilatación progresiva del trayecto creado para colocar la camisa de acceso y de trabajo. Pese a la seguridad de este procedimiento, se ha observado un mayor tiempo de exposición a rayos $X$ (fluoroscopía) tanto del paciente como del personal de salud, así como mayores pérdidas hemáticas. El abordaje realizado en un paso pareciera ser más traumático para alcanzar las dimensiones necesarias para colocar la camisa de acceso en un solo acto, pero se ha observado que reduce el tiempo de exposición a la fluoroscopía y la tasa de complicaciones ${ }^{7}$. El objetivo del estudio fue determinar si existe beneficio del procedimiento en un paso en pacientes con litiasis renal en nuestro centro hospitalario.

\section{Métodos}

El comité local de investigación y ética en salud de la Unidad Médica de Alta Especialidad, Hospital de Especialidades, Centro Médico Nacional de Occidente, del Instituto Mexicano del Seguro Social, aprobó el protocolo de investigación con el registro R-2015-1301-107. Todos los pacientes otorgaron su consentimiento de información debidamente firmado.

Fue un ensayo clínico controlado, ciego simple, realizado en el Departamento de Urología del Hospital de Especialidades Centro Médico Nacional de Occidente en pacientes mayores de 18 años con urocultivo negativo, urocultivo positivo sin urosepsis, actividad de protrombina mayor del $80 \%$, con litiasis compleja no complicada y con función renal estable, aún con tratamiento sustitutivo. Se excluyeron pacientes con urosepsis, con alteraciones en la función respiratoria, insuficiencia cardíaca e insuficiencia renal no controlada.

Se realizó la división de pacientes en dos grupos al azar, mediante elección de sobre cerrado, asignando el número 1 para los pacientes en el grupo control (GC) y el número 2 para los pacientes en el grupo de estudio (GE).

Se midió el tiempo de exposición a rayos $X$ durante la NLP (cronometrado en segundos), así como determinando las variaciones en los valores de hemoglobina.

Se tomaron muestras sanguíneas de cada paciente para biometría hemática completa y concentraciones séricas de creatinina una hora antes de la cirugía.

Los requisitos para la programación quirúrgica fueron contar con biometría hemática dentro de parámetros normales, y si el paciente tenía falla renal era necesario que se encontrara en adecuadas condiciones para la cirugía en cuanto a función cardiaca, respiratoria, etc. A todos los pacientes se les solicitó urocultivo. Una vez ingresados, se inició tratamiento profiláctico con antibiótico (cefalotina, $1 \mathrm{~g}$ por vía intravenosa, o ciprofloxacino, $500 \mathrm{mg}$, en caso de alergia), el cual fue dirigido según los resultados del urocultivo. Al terminar la cirugía se llevó al paciente a recuperación, en donde se llevaron a cabo cuidados posquirúrgicos, y una vez completadas las 24 horas posquirúrgicas se tomaron muestras sanguíneas para biometría hemática, creatinina y urea.

Bajo anestesia general y con antibioticoterapia profiláctica, se colocó al paciente en posición de litotomía cistoscopia con equipo Storz $21 \mathrm{Fr}$, se colocó guía a uréter y catéter de oclusión ureteral, y se administraron $20 \mathrm{ml}$ de material de contraste para 
pielografía por fluoroscopía. Posteriormente se insufló $1 \mathrm{ml}$ de aire al catéter para ubicarlo en la unión ureteropiélica y se posicionó al paciente en decúbito prono para la realización de punción con aguja de nefrostomía en el cáliz seleccionado bajo guía fluoroscópica; se colocó la guía extra rígida y se realizó la dilatación fascial con dilatador de poliuretano 8-14 Fr con guía fluoroscópica, para después reemplazarlo por guía Alken y colocar a través de esta un dilatador Amplatz de calibre $30 \mathrm{Fr}$ de un solo paso al sistema colector. Una vez realizado el trayecto se colocó una camisa de acceso, se realizó nefroscopía con nefroscopio Storz 26 Fr, se localizó el lito y se realizó litotripsia hasta su fragmentación, para posteriormente realizar litolapaxia y extracción con pinza o canastilla. Se realizó nueva nefroscopía, se extrajo el equipo y, en caso de estar indicado, se colocó una sonda de nefrostomía con sonda Foley de dos vías y calibre $22 \mathrm{Fr}$; se insufló un globo con material de contraste y se localizó con fluoroscopia.

Pasadas 24 horas posquirúrgicas se tomaron muestras para biometría hemática, creatinina y urea, y se realizó una placa simple de abdomen en caso de haber empleado acceso supracostal. A todos los pacientes se les realizó, a las 24-48 horas posquirúrgicas, pielografía descendente en caso de contar con nefrostomía.

El error $\alpha$ se fijó en 0.05 , el error $\beta$ en 0.20 y el valor $\delta$ en 11.2 (media y desviación estándar para el tiempo de fluoroscopía en un paso: $41.2 \pm 9.8$; media y desviación estándar para el tiempo de fluoroscopía en abordaje secuencial: $52.4 \pm 10.3$ ). Se estableció una $\mathrm{n}=18$ pacientes en cada grupo para considerarse adecuado, de acuerdo con la fórmula de diferencia de medias para ensayos clínicos basándose en el tiempo de fluoroscopía de Dehong, et al. ${ }^{8}$ (cuatro ensayos clínicos aleatorizados con un total de 6820 pacientes) y Li, et al. ${ }^{9}$ (cuatro ensayos clínicos aleatorizados con un total de 346 pacientes).

La fase descriptiva se realizó con la obtención de números crudos, porcentajes y medidas de tendencia central y dispersión (media \pm desviación estándar). La fase analítica se realizó con prueba exacta de Fisher y ji al cuadrado para las variables cualitativas. Para las cuantitativas se utilizó la prueba t de Student o la prueba $U$ de Mann-Whitney si la distribución de los resultados fue normal o anormal, respectivamente. Todo valor de $p<0.05$ fue considerado estadísticamente significativo. El análisis se realizó con el paquete estadístico SPSS para Windows, versión 20 (IBM Corp., Armonk, NY, USA).

\section{Resultados}

Se dividieron los pacientes en dos grupos: el GE con técnica en un paso $(n=30)$ y el GC con técnica secuencial $(n=29)$. El GE estuvo compuesto por 15 hombres y 15 mujeres, con un promedio de edad de 49.73 años, y el GC por 14 hombres y 15 mujeres, con un promedio de edad de 45.44 años. Las características generales y las variables preoperatorias están descritas en la tabla 1.

EI GE presentó un menor descenso posoperatorio en los valores de hemoglobina que el GC, siendo estadísticamente significativo (-0.81 vs. $-2.03 \mathrm{~g} / \mathrm{dl}$; $p<0.001)$. La misma tendencia se observó en los valores de creatinina, donde se mostró una disminución en el GE y un aumento en el GC, siendo la diferencia también significativa $(-0.05$ vs. $+0.19 \mathrm{mg} / \mathrm{dl}$; $p<0.001)$. No obstante, no se observó deterioro en la función renal. Asimismo, en los tiempos de exposición a radiación ionizante se encontraron tiempos significativamente menores en el GE que en el GC (69.6 vs. $100.62 \mathrm{~s} ; \mathrm{p}<0.001)$. Las variables operatorias evaluadas se encuentran detalladas en la tabla 2. Los días de estancia intrahospitalaria para el GE fueron, en promedio, 2.23 días, y en el GC fueron 2.34 días ( $p<0.42)$.

En cuanto a complicaciones, un paciente de cada grupo requirió transfusión sanguínea, un paciente del GC presentó fístula pleural secundaria a un absceso supracostal y ningún paciente tuvo hemorragia grave, hematoma renal ni requirió otro tipo de intervención. No hubo lesiones viscerales o vasculares, ni infección de la herida quirúrgica. De la misma manera, la respuesta inflamatoria sistémica, como signos de taquicardia $(6 \%$ de los pacientes del GC), fiebre ( $6 \%$ de los pacientes de ambos grupos) o leucocitosis (33\% de los pacientes del GE y $31 \%$ del GC), así como la necesidad de ser reintervenidos quirúrgicamente (ningún paciente), no mostraron diferencias significativas (Tabla 3).

De acuerdo con la clasificación de Clavien-Dindo para complicaciones quirúrgicas, que se divide en grados del I al V según la gravedad de las complicaciones y el tratamiento que requieren, se clasificaron los pacientes de la siguiente manera: en el GC hubo dos pacientes con fiebre, dos con taquicardia y nueve con leucocitosis (grado I), un paciente que requirió transfusión (grado II) y un paciente con una fístula pleural (grado IIla); en el GE hubo dos pacientes con 
Gaceta Médica de México. 2017;153

Tabla 1. Características basales de los pacientes

\begin{tabular}{|c|c|c|c|}
\hline Variable & $\begin{array}{l}\text { Técnica en un paso } \\
\qquad(\mathrm{N}=30)\end{array}$ & $\begin{array}{l}\text { Técnica secuencial } \\
\qquad(\mathrm{N}=29)\end{array}$ & $\mathrm{p}$ \\
\hline $\mathrm{IMC}\left(\mathrm{kg} / \mathrm{m}^{2} \pm \mathrm{DE}\right)$ & $28.04 \pm 5.49$ & $27.45 \pm 4.30$ & 0.74 \\
\hline Media de edad (años \pm DE) & $49.73 \pm 14.3$ & $45.44 \pm 14.4$ & 0.31 \\
\hline Sexo masculino & 15 & 15 & $0.89^{*}$ \\
\hline Sexo femenino & 15 & 14 & \\
\hline Nefrolitotomía previa & 5 & 6 & $0.16^{*}$ \\
\hline Riñón en herradura & 2 & 3 & $0.48^{*}$ \\
\hline Tamaño del cálculo (cm) & $2.23 \pm 1.15$ & $2.52 \pm 1.14$ & 0.34 \\
\hline Riñón izquierdo & 14 & 16 & $0.51^{*}$ \\
\hline Riñón derecho & 16 & 13 & \\
\hline Cálculo en cálices superiores & 3 & 2 & $0.90^{*}$ \\
\hline Cálculo en cálices medios & 15 & 14 & \\
\hline Cálculo en cálices inferiores & 8 & 10 & \\
\hline Cálculo coraliforme & 4 & 3 & \\
\hline Hemoglobina (g/dl) & $14.33 \pm 2.08$ & $14.64 \pm 0.96$ & 0.23 \\
\hline Creatinina sérica (mg/dl) & $0.98 \pm 0.42$ & $0.93 \pm 0.37$ & 0.71 \\
\hline Acceso por un trayecto & 27 & 24 & $0.33^{*}$ \\
\hline Acceso por dos trayecto & 3 & 5 & \\
\hline
\end{tabular}

DE: desviación estándar; IMC: índice de masa corporal.

Tabla 2. Resultados de laboratorio preoperatorios y posoperatorios, tiempo de fluoroscopía y tasa de transfusión sanguínea

\begin{tabular}{lccc}
\hline Variable & $\begin{array}{c}\text { Técnica en un paso } \\
(\mathbf{N}=\mathbf{3 0})\end{array}$ & $\begin{array}{c}\text { Técnica secuencial } \\
(\mathbf{N}=\mathbf{2 9})\end{array}$ & $\mathbf{p}$ \\
\hline Tiempo de exposición a fluoroscopía $(\mathrm{s} \pm \mathrm{DE})$ & $69.60 \pm 21.38$ & $100.62 \pm 23.54$ & $<0.001^{*}$ \\
Hemoglobina preoperatoria $(\mathrm{g} / \mathrm{dl} \pm \mathrm{DE})$ & $14.33 \pm 2.08$ & $14.64 \pm 0.96$ & $0.47^{*}$ \\
Hemoglobina posoperatoria $(\mathrm{g} / \mathrm{dl} \pm \mathrm{DE})$ & $13.52 \pm 1.79$ & $12.61 \pm 1.78$ & $0.03^{*}$ \\
Diferencia hemoglobina pre/post $(\mathrm{g} / \mathrm{dl} \pm \mathrm{DE})$ & $0.81 \pm 0.78$ & $2.03 \pm 1.04$ & $<0.001^{*}$ \\
Creatinina preoperatoria $(\mathrm{mg} / \mathrm{dl} \pm \mathrm{DE})$ & $0.98 \pm 0.42$ & $0.93 \pm 0.37$ & $0.62^{*}$ \\
Creatinina posoperatoria $(\mathrm{mg} / \mathrm{dl} \pm \mathrm{DE})$ & $0.93 \pm 0.29$ & $1.13 \pm 0.43$ & $0.039^{*}$ \\
Diferencia de creatinina sérica $(\mathrm{mg} / \mathrm{dl} \pm \mathrm{DE})$ & $-0.055 \pm 0.071$ & $3.57 \%$ & $<0.001^{*}$ \\
Tasa de transfusión sanguínea & $3.44 \%$ & & $0.74^{\dagger}$ \\
\hline
\end{tabular}

${ }^{*}$ Calculada por t de Student.

${ }^{\dagger}$ Calculada por prueba exacta de Fisher.

DE: desviación estándar.

fiebre, diez con leucocitosis (grado I) y uno que requirió transfusión (grado II).

\section{Discusión}

La NLP ha venido a cambiar el uso de la cirugía abierta por un procedimiento mínimamente invasivo, reduciendo las complicaciones para el paciente, así como los días de estancia hospitalaria ${ }^{10}$. Aun con los avances en la técnica quirúrgica, los pacientes y el personal de salud tienen que pasar por periodos quirúrgicos y tiempos de exposición a radiación prolongados.

La técnica de dilatación con balón es el método mejor aceptado para realizar el trayecto en cirugía percutánea, pero su alto costo limita su uso de forma 
Tabla 3. Mortalidad y morbilidad posoperatoria

\begin{tabular}{|c|c|c|c|}
\hline Variable & $\begin{array}{l}\text { Técnica en } \\
\text { un paso } \\
(\mathrm{N}=30)\end{array}$ & $\begin{array}{l}\text { Técnica } \\
\text { secuencial } \\
(\mathrm{N}=29)\end{array}$ & p \\
\hline Estancia hospitalaria (días) & $2.23 \pm 0.56$ & $2.34 \pm 0.48$ & $0.42^{\star}$ \\
\hline \multicolumn{4}{|l|}{ Nefrostomía } \\
\hline Sí & 24 & 25 & $0.52^{\dagger}$ \\
\hline No & 6 & 4 & \\
\hline \multicolumn{4}{|l|}{ Fiebre } \\
\hline Sí & 2 & 2 & $0.51^{\ddagger}$ \\
\hline No & 28 & 27 & \\
\hline \multicolumn{4}{|l|}{ Leucocitosis } \\
\hline Sí & 10 & 9 & $0.85^{\dagger}$ \\
\hline No & 20 & 20 & \\
\hline \multicolumn{4}{|l|}{ Taquicardia } \\
\hline Sí & 0 & 2 & $0.23^{\ddagger}$ \\
\hline No & 30 & 27 & \\
\hline \multicolumn{4}{|l|}{ Transfusión } \\
\hline Sí & 1 & 1 & $0.74^{\ddagger}$ \\
\hline No & 29 & 28 & \\
\hline \multicolumn{4}{|l|}{ Lesión a órganos vecinos } \\
\hline Sí & 0 & 2 & $0.23^{\ddagger}$ \\
\hline No & 30 & 28 & \\
\hline \multicolumn{4}{|l|}{ Complicaciones } \\
\hline Sí & 1 & 1 & $0.74^{\ddagger}$ \\
\hline No & 29 & 28 & \\
\hline
\end{tabular}

sistemática; sin embargo, el estudio realizado por Yamaguchi, et al. ${ }^{7}$ asocia la dilatación con balón con un mayor tiempo quirúrgico, lo que conlleva mayor sangrado. La dilatación de Amplatz o con dilatadores metálicos aumenta el tiempo de exposición a radiación y consume mayor tiempo transoperatorio. La razón por la cual la técnica en un paso ha aumentado su aceptación entre los urólogos es su bajo costo, así como un tiempo quirúrgico y una duración de la fluoroscopía reducidos ${ }^{7-9}$.

Frattini, et al. ${ }^{11}$ condujeron un estudio con 78 pacientes en el que compararon la dilatación secuencial, la técnica en un paso y la dilatación con balón en cuanto a exposición a rayos $X$, pérdida sanguínea y costo-beneficio. Sus resultados arrojan que la técnica en un paso es comparable con las otras dos técnicas de dilatación, debido a que no presentó un aumento en la morbilidad y redujo de manera significativa la exposición a radiación y los costos, y además tuvo una menor tasa de hemorragia en comparación con las otras dos. En nuestro estudio no se evaluaron los costos del material, pero esta podría ser una variable importante a favor de la utilización de la técnica en un paso, ya que solo se requiere un único dilatador desechable abocado en guía Alken reusable, reduciendo aún más los costos.

Una complicación muy importante asociada con la cirugía renal percutánea es la hemorragia. En el estudio de Stoller, et al. ${ }^{12}$ se evaluaron factores asociados a pérdida de sangre en la NLP en 127 pacientes y no se encontró un aumento en el riesgo de hemorragia utilizando dilatadores fasciales ${ }^{12}$. En nuestro estudio se utilizó dilatación fascial con calibres 8-14 Fr en todos los casos, sin que esto se asociara a hemorragia del trayecto. Además, este tipo de dilatación puede mejorar el acceso en pacientes con cirugía previa.

Varios estudios han comparado directamente la técnica en un paso con la dilatación secuencial utilizando Amplatz o dilatadores metálicos. Se ha demostrado que la dilatación en un paso tiene un menor requerimiento de transfusiones sanguíneas ${ }^{5,6,10}$. Nuestro estudio evalúo la pérdida sanguínea con la determinación de los valores séricos de hemoglobina antes y después de la intervención, encontrando un descenso significativo en los pacientes que se sometieron a dilatación en un paso frente a la dilatación secuencial $(p<0.001)$. Aunque estos resultados son estadísticamente significativos, no se encontró diferencia en el número de transfusiones sanguíneas entre ambos grupos $(p=0.74)$.

Aminsharifi, et al..$^{13}$ condujeron un estudio que reveló el daño causado al parénquima renal comparando la dilatación en un paso contra la dilatación secuencial a través de la captación de tecnecio 99 en una gammagrafía con ácido dimercaptosuccínico (DMSA), encontrando que 14 de 29 pacientes con la técnica en un paso presentaron una nueva cicatriz en el parénquima, en comparación con 2 de 29 pacientes del grupo de dilatación secuencial. En nuestro estudio se pretendió medir de manera indirecta la función renal con los valores séricos de creatinina; sin embargo, tanto la captación de tecnecio 99 en la gammagrafía con DMSA como los valores séricos de creatinina no son la modalidad diagnóstica de elección para determinar la función renal. Se requieren mejores estudios para determinar el daño renal a corto y largo plazo; no obstante, nuestros hallazgos en los valores de creatinina sérica sugieren que la técnica en un paso es más segura en términos de preservación de la función renal, pero no para disminuir la cicatrización renal. A pesar de haber obtenido resultados estadísticamente significativos con 
respecto a los valores de hemoglobina y creatinina, no se consideran clínicamente significativos porque en ningún grupo hubo daño renal ni deterioro de la función renal.

Frattini, et al. ${ }^{11}$ refieren la hipermotilidad renal y la cirugía previa como contraindicaciones relativas de la técnica en un paso, porque aumentan la tasa de complicaciones asociadas, como daño vascular o en estructuras adyacentes. Cuando se identifica alguno de estos factores de riesgo se recomienda realizar el trayecto de nefrostomía con la técnica convencional ${ }^{11}$. Con el fin de reducir estas complicaciones, la fuerza radial aplicada es crucial, utilizando movimientos rotacionales durante el proceso gradual para reducir así el desplazamiento de la unidad renal y la tasa de falla a un $7 \%$ en pacientes con cirugía previa $^{8,13,14}$.

Dentro de las limitaciones al realizar este estudio encontramos las dificultades para la evaluación de la función renal de manera directa. A pesar de que la experiencia del cirujano influye en el resultado de toda cirugía, no consideramos una limitante que los procedimientos realizados para este estudio hayan sido realizados por un solo cirujano.

En nuestro estudio se encontraron cinco pacientes en el GE y seis en el GC con cirugía renal previa, pero fue posible realizar la cirugía sin ninguna dificultad en todos ellos y no se asoció con un aumento en la tasa de hemorragia ni en el daño a estructuras vecinas. Únicamente un paciente del GE presentó hipermotilidad renal durante el procedimiento, haciendo imposible la realización de la técnica en un paso, lo que se asoció a un aumento en el tiempo de fluoroscopía y la dilatación tuvo que ser completada de manera secuencial sin complicaciones. Por lo tanto, se recomienda la dilatación secuencial en aquellos casos en los que se detecte hipermotilidad renal.

Un hallazgo importante en este estudio fue que el cirujano que realizó los procedimientos notó que el tipo de dilatación afectaba el campo visual durante la cirugía. Esto se asoció a hemorragia transquirúrgica, la cual fue mayor en el GC. Sin embargo, esta variable no fue medible. Se deben llevar a cabo más estudios sobre este tema en particular, debido a la limitada literatura acerca del problema, así como estudios acerca de la rentabilidad de esta técnica y sobre todo en relación con el beneficio y la preservación de la función renal medida de manera específica.

\section{Conclusiones}

La técnica en un paso es más segura y efectiva para realizar el trayecto de nefrostomía en los pacientes con litiasis renal independientemente del tamaño, la localización, los antecedentes y la variación anatómica, sin aumentar las tasas de complicaciones y reduciendo considerablemente los tiempos de radiación y la cantidad de hemorragia.

Se recomienda el uso de la técnica en un paso en pacientes con nefrolitiasis que son candidatos para NLP, por los beneficios que confiere al paciente y al personal de salud.

\section{Conflictos de intereses}

Los autores declaran que no existe ningún conflicto de intereses. Todos los autores aprobaron la versión final del manuscrito.

\section{Financiamiento}

No requirió financiamiento.

\section{Bibliografía}

1. Wein K. Litiasis urinaria: etiología, diagnóstico y manejo médico. En: Wein A, Kavoussi L, Novick A, et al., editores. Campbell-Walsh. Urología. 10. a ed. Philadelphia: Saunders Elsiever; 2010. p. 1257-410.

2. Middela S, Papadopoulos G, Srirangam S, et al. Extracorporeal shock wave lithotripsy for ureteral stones: do decompression tubes matter? Urology. 2010;76:821-5.

3. Türk C, Knoll T, Köhrmann KU. New guidelines for urinary stone treatment. Controversy or development? Urologe A. 2008;47:591-3.

4. Wen CC, Nakada SY. Treatment selection and outcomes: renal calculi. Urol Clin North Am. 2007;34:409-19.

5. Castillo O, Pinto I, Díaz M, et al. Cirugía percutánea de litiasis coraliforme. Rev Chil Cirug. 2008;60:393-7.

6. Michel MS, Trojan L, Rassweiler JJ. Complications in percutaneous nephrolitotomy. Eur Urol. 2007:51:899-906.

7. Yamaguchi A, Skolarikos A, Buchholz NP, et al. Clinical Research Office of the Endourological Society Percutaneous Nephrolithotomy Study Group. Operating times and bleeding complications in percutaneous nephrolithotomy: a comparison of tract dilation methods in 5,537 patients in the Clinical Research Office of the Endourological Society Percutaneous Nephrolithotomy Global Study. J Endourol. 2011;25:933-9.

8. Dehong $C$, Liangren $L$, Huawei $L$, et al. A comparison among four tract dilation methods of percutaneous nephrolithotomy: a systematic review and meta-analysis. Urolithiasis. 2013;41:523-30.

9. Li Y, Yang L, Xu P, et al. One-shot versus gradual dilation technique for tract creation in percutaneous nephrolithotomy: a systematic review and meta-analysis. Urolithiasis. 2013;41:443-8.

10. Deem S, DeFade B, Modak A, et al. Percutaneous nephrolithotomy versus extracorporeal shock wave lithotripsy for moderate size kidney stones. Urology. 2011;78:739-43.

11. Frattini A, Barbieri A, Salsi $P$, et al. One-shot: a novel method to dilate the nephrostomy access for percutaneous lithotripsy. J Endourol. 2001;15:919-23.

12. Stoller ML, Wolf JS Jr, St Lezin MA. Estimated blood loss and transfusion rates associated with percutaneous nephrolithotomy. J Urol. 1994:152:1977-81.

13. Aminsharifi A, Alavi M, Sadeghi G, et al. Renal parenchymal damage after percutaneous nephrolithotomy with one-stage tract dilation technique: a randomized clinical trial. J Endourol. 2011;25:927-31.

14. Amjadi M, Zolfaghari A, Elahian A, et al. Percutaneous nephrolithotomy in patients with previous open nephrolithotomy: one-shot versus telescopic technique for tract dilatation. J Endourol. 2008;22:423-5. 